\title{
Um estudo sobre gestão do conhecimento em escritórios de gerenciamento de projetos na região metropolitana de Belo Horizonte
}

\section{A study on knowledge management in project management offices in the metropolitan region of Belo Horizonte}

\author{
Vladimir Alexei Rodrigues Rocha \\ Doutorando em Ciência da Informação pela Universidade Federal de Minas Gerais \\ Ricardo Rodrigues Barbosa \\ Doutorado em Administração por Columbia University \\ Docente da Escola de Ciência da Informação da Universidade Federal de Minas Gerais \\ George Leal Jamil \\ Doutor em Ciência da Informação pela Universidade Federal de Minas Gerais \\ Pesquisador convidado do InescTec
}

\begin{abstract}
Resumo: A Gestão do Conhecimento, em um cenário competitivo como o atual, é uma necessidade e um desafio para as organizações. Este estudo teve como objetivo refletir em como a gestão do conhecimento é compreendida em Escritórios de Gerenciamento de Projetos (EGP). Justificou-se em função do cenário e em razão da originalidade e temporalidade dos projetos. De natureza descritiva, utilizou-se como método a análise de múltiplos casos, por julgamento não probabilístico no EGP de quatro organizações brasileiras, de segmentos diferentes. Os resultados apontaram para oito conhecimentos classificados como relevantes, cinco fatores influenciadores que facilitam e quatro que dificultam o processo de gestão do conhecimento. Além disso, foram mapeadas práticas de gestão do conhecimento entre projetos e no plano do projeto. Concluiu-se que o contexto organizacional favorável, isto é, com processos estruturados e colaboradores capacitados, seria um dos pontos principais da gestão do conhecimento em EGP e um dos seus maiores desafios.
\end{abstract}

Palavras-chave: Gestão do Conhecimento. Gestão de Projetos. Escritório de Gerenciamento de Projetos.

\begin{abstract}
Knowledge Management, in a competitive scenario like the current one, is a necessity and a challenge for organizations. This study aimed to reflect on how knowledge management is understood in Project Management Offices (PMO). It was justified according to the scenario and due to the originality and temporality of the projects. Of a descriptive nature, the analysis of multiple cases was used as a method, by non-probabilistic judgment in the EGP of four Brazilian organizations, from different segments. The results pointed to eight knowledge classified as relevant, five influencing factors that facilitate and four that hinder the knowledge management process. In addition, knowledge management practices were mapped between projects and at the project plan. It was concluded that the favorable organizational context, that is, with structured processes and trained employees, would be one of the main points of knowledge management in EGP and one of its greatest challenges.
\end{abstract}

Keywords: Knowledge management. Project management. Project Management Office. 


\section{IRIS)}

Um estudo sobre gestão do conhecimento em escritórios de gerenciamento de projetos na região metropolitana de Belo Horizonte Vladimir Alexei Rodrigues Rocha, Ricardo Rodrigues Barbosa, George Leal Jamil

\section{Introdução}

A sociedade contemporânea passa por profundas transformações e com as organizações não tem sido diferente. No contexto econômico essa mudança também foi percebida, quando houve a transformação de uma economia de bens, para uma economia baseada em conhecimento, segundo Peter Drucker (1995). Na era do conhecimento, o patrimônio indispensável para as empresas deixou de ser a indústria e o equipamento, e passou a ser o conhecimento acumulado da organização e de seus profissionais.

A transformação da economia de bens para uma economia baseada em conhecimento, apresenta mudança de paradigma nas organizações, pois a fonte de poder muda do nível hierárquico ocupado, para o nível do conhecimento obtido (SVEIBY, 1997). A mudança nas organizações pode ser percebida sob a perspectiva de "pessoas", quando o paradigma deixa de ser "pessoas como geradores de custos ou recursos" (era industrial), e passa a ser percebido como "geradores de receitas" (era do conhecimento). Outra mudança ocorre na "produção", quando o paradigma deixa de ser "operários processando recurso físico para criar produtos tangíveis", e passa para "trabalhadores do conhecimento convertendo conhecimento em estruturas intangíveis". E por fim, "clientes", quando a relação com o cliente deixa de ser "unidirecional via mercado" e passa a ser “interativa, via rede de pessoas” (SVEIBY, p. 27, 1997).

Por sua importância econômica, acadêmicos e profissionais de mercado passaram a estudar formas de gerenciar o conhecimento nas organizações, desenvolvendo, a partir deste ponto, o campo de estudo da Gestão do Conhecimento (GC). A OCDE (Organization for Economic Cooperation and Development), reconhece a "economia baseada em conhecimento" e a sua importância para os países membros, como sendo a economia ligada diretamente à produção, distribuição e uso do conhecimento e informação (MÊGNIGBÊTO, 2018).

O conhecimento produzido e acumulado nas organizações, requer gestão, do contrário se perde. Para Nonaka e Takeuchi (2008), a gestão desse conhecimento pode ser definida como o processo de criar continuamente novos conhecimentos, disseminando-os amplamente na organização e incorporando-os velozmente em produtos/serviços, tecnologias e sistemas. Spender (2008) complementa que a Gestão do Conhecimento está relacionada à otimização do valor econômico entregue, realizando o potencial econômico do conhecimento. Dessa forma, o conhecimento gera possibilidades de melhoria nos resultados do negócio. 
Para desenvolver produtos e serviços em uma economia em transformação, algumas organizações adotaram como ferramenta a gestão de projetos. Serrat (2017) trabalha projeto como um modelo lógico, aplicável em diversos segmentos de negócios, com o objetivo de fortalecer o design e proporcionar melhor monitoramento das atividades em suas diversas etapas, como impulsionadores do processo de mudança organizacional. Projeto, segundo o Project Management Institute [PMI (2017), é um empreendimento temporário para criar um produto, serviço ou resultado exclusivo. Contém a noção de temporalidade - começo e fim - e de originalidade - único, exclusivo.

O PMI é uma das diversas associações existentes, com o objetivo de promover as melhores práticas em gerenciamento de projetos. Sua produção mais conhecida é o Guia PMBOK - Project Management Body of Knowledge (Um Guia do Conhecimento em Gerenciamento de Projetos). As quatro organizações pesquisadas, adotam, de acordo com a necessidade, integral ou parcialmente o conjunto de melhores práticas difundidos pelo PMI.

A gestão do conhecimento é importante para o PMI pois, uma das alterações da sexta edição do Guia PMBOK (PMI, 2017), foi a inclusão de três novos processos, sendo um deles gerenciar o conhecimento do projeto, um campo relativamente novo de estudos (HORSTEIN, 2014). Os outros dois são implementar respostas aos riscos e controlar recursos. Cabe, ao Escritório de Gerenciamento de Projetos (EGP), o papel da gestão do conhecimento produzido na gestão de projetos (AUBRY et al., 2011; MÜLLER et al., 2013; NADAE; CARVALHO, 2017).

Como conhecimento é algo de difícil estruturação (DAVENPORT \& PRUSAK, 1998), seu funcionamento no contexto em que as organizações lidam com projetos como ferramenta de gestão, é ainda mais desafiador, por isso a pergunta que motivou o estudo foi entender, como é realizada a gestão do conhecimento em escritórios de gerenciamento de projetos?

Assim sendo, em função das mudanças de paradigmas - economia industrial para economia do conhecimento -, da natureza dos projetos - temporalidade e originalidade - e do contexto das organizações, a presente pesquisa teve como objetivo geral investigar como ocorre a gestão do conhecimento em Escritórios de Gerenciamento de Projetos (EGP), desenvolvidos em organizações brasileiras de segmentos diferentes. Embora, ao explorar segmentos diferentes é possível que particularidades não sejam contempladas e, com isso, incorrer no risco da superficialidade. Adotouse como objetivos específicos, (i) mapear conhecimentos relevantes em projetos, (ii) identificar 
Um estudo sobre gestão do conhecimento em escritórios de gerenciamento de projetos na região metropolitana de Belo Horizonte Vladimir Alexei Rodrigues Rocha, Ricardo Rodrigues Barbosa, George Leal Jamil

práticas de gestão do conhecimento em projetos, e (iii) fatores influenciadores que facilitam e dificultam as práticas de gestão do conhecimento em projetos.

Justifica-se a pesquisa como uma contribuição acadêmica para o campo da Gestão do Conhecimento em Projetos, a partir do mapeamento dos conhecimentos considerados relevantes para a execução dos projetos, identificação de práticas que viabilizam a gestão do conhecimento entre projetos e no próprio projeto a partir da gestão do conhecimento em um escritório de gerenciamento de projetos. Do ponto de vista prático, justifica-se quando aponta fatores facilitadores e dificultadores para a gestão do conhecimento, de forma a que se possa investir esforços nas organizações para ampliar os pontos facilitadores e mitigar aqueles dificultadores que tornam o processo mais lento ou com resultados inferiores ao que se espera para o tratamento adequado do conhecimento.

\section{Revisão de literatura}

Discute-se, nesta seção, a literatura relacionada com o tema da pesquisa, estruturada em quatro subtópicos, que são o conhecimento (2.1), gestão do conhecimento, processos, projetos, práticas e fatores influenciadores (2.2), gestão do conhecimento em escritórios de gerenciamento de projetos (2.3) e, por fim, estudos anteriores (2.4).

\subsection{Conhecimento}

Conhecimento é informação que faz com que o indivíduo ou organização haja de forma diferente ou mais eficaz, de acordo com Peter Drucker (2011).

Nonaka e Takeuchi (1997), tratam o conceito de conhecimento, como "crença verdadeira justificada", desenvolvida por Platão. Os autores ainda argumentam que o Ocidente concentrou a definição na "verdade" enquanto eles trabalharam a "crença justificada".

Para efeito de entendimento nessa pesquisa, podem ser observadas as seguintes definições na Tabela 1: 
Tabela 1 - Definições de conhecimento

\begin{tabular}{|l|l|}
\hline \multicolumn{1}{|c|}{ DEFINIÇÕES } & \multicolumn{1}{|c|}{ REFERÊNCIAS } \\
\hline $\begin{array}{l}\text { Conjunto obtido pela informação e o contexto associado, } \\
\text { envolvendo a percepção do ambiente e do sistema }\end{array}$ & $\begin{array}{l}\text { Nonaka e Takeuchi, } \\
1997 ; \text { Alle, 1997; } \\
\text { Davenport, 1998. }\end{array}$ \\
\hline $\begin{array}{l}\text { Informação processada tendo em vista o cumprimento de } \\
\text { determinado propósito }\end{array}$ & $\begin{array}{l}\text { Faucher, Everett e } \\
\text { Lawson, 2008. }\end{array}$ \\
\hline $\begin{array}{l}\text { Diz respeito a crenças e compromissos, função direta de uma } \\
\text { atitude, perspectiva ou intenção específica com algum fim }\end{array}$ & $\begin{array}{l}\text { Sampaio, Rosa e Pereira, } \\
2012 .\end{array}$ \\
\hline $\begin{array}{l}\text { Capturar a experiência de conhecimento coletivo da } \\
\text { organização, distribuindo-o para obtenção de vantagem } \\
\text { competitiva }\end{array}$ & $\begin{array}{l}\text { Ochieng e Nyangosi, } \\
2017 .\end{array}$ \\
\hline $\begin{array}{l}\text { Processo humano dinâmico de justificar crença pessoal em } \\
\text { relação à verdade }\end{array}$ & $\begin{array}{l}\text { Besen, Tecchio e Fialho, } \\
2017 .\end{array}$ \\
\hline $\begin{array}{l}\text { Um tipo especial de estado mental do indivíduo edificado a } \\
\text { partir da absorção de informações que regem as ações do } \\
\text { próprio indivíduo }\end{array}$ & Trevisan e Damian, 2018. \\
\hline $\begin{array}{l}\text { O uso ideal relacionado ao trabalho, experiência e habilidades } \\
\text { dos funcionários }\end{array}$ & $\begin{array}{l}\text { Haraldsdottir, } \\
\text { Gunnlaugsdottir, } \\
\text { Hvannberg e Christensen, } \\
2018 .\end{array}$ \\
\hline
\end{tabular}

Fonte: autores, 2020.

Santos, Isaton e Jungles (2015) lembram ainda que conhecimento não é algo linear e sim transmitido de um colaborador para outro, avançando em conjunto, levando ideias, valores e emoções. O que pode ser percebido também em projetos.

O conhecimento, no presente trabalho, segue a linha de pensamento de Nonaka e Takeuchi (1997), quando compreende ser uma "crença justificada", que pode ser modificado com base em experiências e de acordo com o contexto organizacional.

Por fim, é possível classificar o conhecimento sob diversos pontos de vista. Pode-se diferenciá-lo por forma de compreender a realidade, como fazem alguns autores em metodologia de pesquisa, como Martins e Theóphilo (2009) e Marconi e Lakatos (2010), ou classificá-lo segundo as diferenças em suas dimensões, como fazem Nakano e Fleury (2005). Ainda é possível tratar sob o ponto da ontologia (individual, grupo, organizacional e interorganizacional) e epistemológica (tácito e explícito), conforme trabalhado por Nonaka e Takeuchi (1997).

\subsection{Gestão do Conhecimento, processos, projetos, práticas e fatores influenciadores}


Um estudo sobre gestão do conhecimento em escritórios de gerenciamento de projetos na região metropolitana de Belo Horizonte Vladimir Alexei Rodrigues Rocha, Ricardo Rodrigues Barbosa, George Leal Jamil

Segundo Jannuzzi, Falsarella e Sugahara (2016), o reconhecimento da importância do conhecimento nas organizações tem contribuído para o desenvolvimento de muitos estudos, inclusive, sobre a gestão do conhecimento. No entanto, continuam os autores, apesar dos esforços, é fato que a gestão do conhecimento não se constitui uma tarefa fácil. Para North (2010), a gestão do conhecimento significa equilibrar a estabilidade e renovação com a cooperação e a competição no ambiente organizacional.

Nonaka e Takeuchi (2008, p. I), apresentam a Gestão do Conhecimento como "o processo de criar continuamente novos conhecimentos, disseminando-os amplamente através da organização e incorporando-os velozmente em projetos/serviços, tecnologias e sistemas - perpetua a mudança no interior da organização".

Dalkir (2005, p. 3) propõe uma definição próxima, mas com um foco nivelado entre criação e reutilização quando define que "Gestão do Conhecimento é a coordenação deliberada e sistêmica das pessoas, tecnologia, processos e estrutura organizacional, de forma a agregar valor por meio da reutilização e inovação".

Nonaka e Takeuchi (1997, p. 67) formularam o modelo SECI - socialização, externalização, combinação e internalização - tendo em vista a criação do conhecimento a partir da interação do conhecimento tácito e explícito. Essa interação é chamada pelos autores de "conversão do conhecimento", que ressalta ser "um processo 'social' entre indivíduos, e não confinada dentro de um indivíduo", conforme Figura 1.

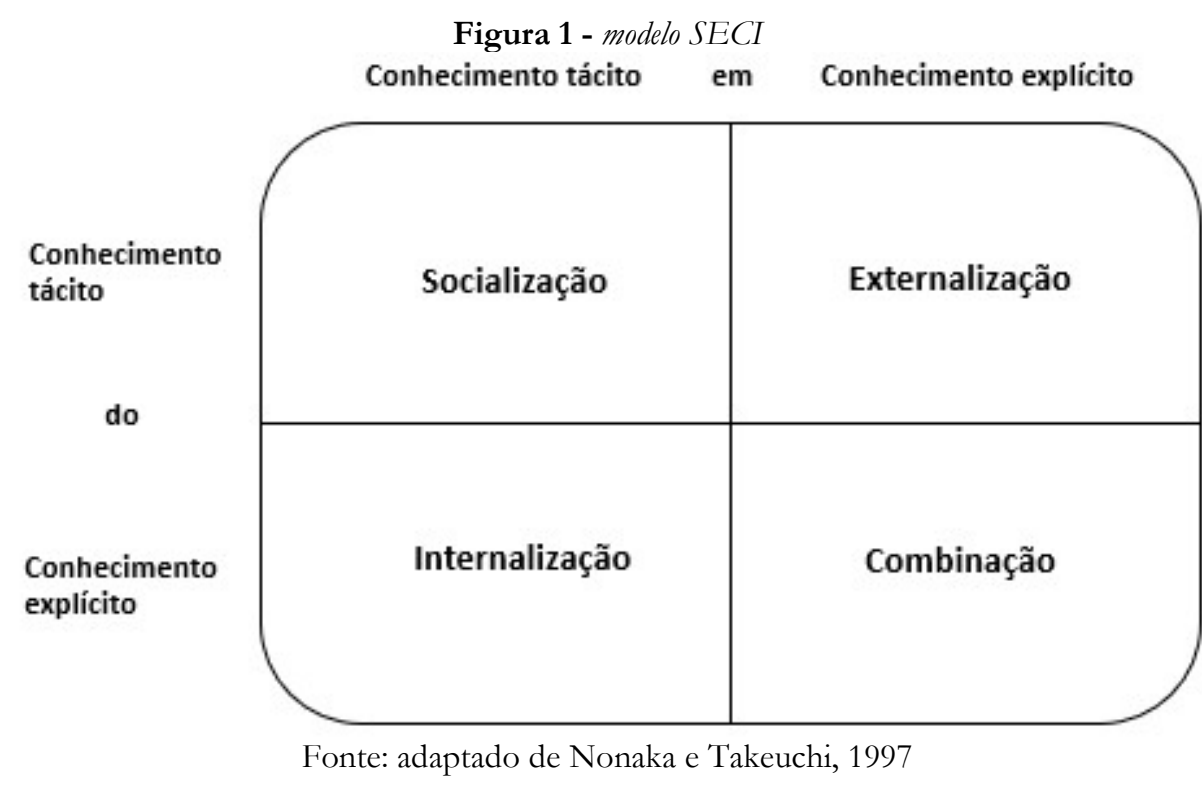


Um estudo sobre gestão do conhecimento em escritórios de gerenciamento de projetos na região metropolitana de Belo Horizonte Vladimir Alexei Rodrigues Rocha, Ricardo Rodrigues Barbosa, George Leal Jamil

$\mathrm{Na}$ Socialização, há transformação do conhecimento tácito em tácito por meio do compartilhamento de experiências e geração do conhecimento na forma de modelos mentais ou habilidades técnicas.

A Externalização é o processo de transformação do conhecimento tácito em explícito. Processo de articulação do conhecimento tácito em conceitos explícitos, sob a forma de metáforas, analogias, hipóteses e modelos.

No processo de Combinação, há a transformação do conhecimento explícito em explícito. Envolve combinar conjuntos diferentes de conhecimento explícito como, por exemplo, documentos, reuniões, conversas ao telefone ou redes de comunicação computadorizadas (NONAKA \& TAKEUCHI, 1997).

Registram ainda, os autores citados, a Internalização, que seria a transformação do conhecimento explícito em tácito. É o processo de incorporar o conhecimento explícito ao conhecimento tácito, intimamente relacionado ao "aprender fazendo".

$\mathrm{Na}$ presente pesquisa, entender a socialização do conhecimento em Escritórios de Gerenciamento de Projetos, torna a gestão do conhecimento um campo de estudo que trata a identificação, processos, práticas e fatores que influenciam - facilitando ou dificultando, a disseminação do conhecimento nas organizações.

Por fim, à medida que as organizações amadurecem, os padrões de interação e comunicação se tornam formalizados, fazendo parte do processo da organização, sem, necessariamente, constar o procedimento como descrito por Nonaka e Takeuchi (1997).

Seguindo com os estudos, para compreender o conhecimento em projetos, preservam-se as definições apresentadas na revisão da literatura, acrescentando a classificação do conhecimento em projetos. De acordo com Hanisch, Lindner, Mueller e Wald (2009), o conhecimento pode ser "em" projetos, "sobre" projetos e "entre" projetos.

Conhecimento em projetos trata da metodologia adotada na gestão de projetos, cuja estrutura é composta por processos de iniciação, planejamento, execução, monitoramento e controle, além dos processos de encerramento (PMI, 2017). Contemplam-se ainda práticas de comunicação em projetos, tanto com equipes alocadas em múltiplas unidades, como em unidade única. Segundo Hanisch et al. (2009), o conhecimento em projetos possui forte influência do gestor do projeto e, consequentemente, do estilo adotado no gerenciamento do projeto. 
Conhecimento sobre projetos é a visão geral do cenário em que os projetos foram ou estão sendo estruturados, de acordo com Hanisch et al. (2009). O cenário é analisado com o objetivo de manter o conhecimento produzido pelos projetos, acessíveis aos gestores. Em função da natureza única e temporária dos projetos, organizar o conhecimento sobre projetos é um dos fatores críticos de sucesso, para evitar o que Bahia (2012) chamou de "amnésia nos projetos". A "perda de memória”, segundo o autor, é destacada em função da alta pressão para o término do projeto e a alocação de recursos já planejados para outros projetos.

Por fim, o conhecimento entre projetos, ainda em Hanisch et al. (2009), é caracterizado pelo conhecimento produzido decorrente da similaridade entre os projetos, conhecimento metodológico, lições aprendidas e conhecimento processual. Para Menezes (2012), o conhecimento entre projetos permite padronização, melhor alocação de recursos, tomada de decisão assertiva quanto a aplicação dos recursos, principalmente naquilo que é conhecido como "tríplice restrição" em projetos (escopo, tempo e custo).

Hanisch et al. (2009) apontam alguns tipos de conhecimentos utilizados nas práticas de gestão do conhecimento, informando ainda que esses conhecimentos variam de acordo com o ciclo de vida do projeto. $\mathrm{Na}$ fase de planejamento a busca por lições aprendidas, conhecimentos técnicos e de clientes são alguns exemplos. Na implementação do projeto, aplicação de conhecimentos sobre planejamento e orçamento, preparação da proposta, busca de documentos de melhores práticas, identificação de especialistas e uso de métodos de gestão de projetos consolidados e melhorados são outros exemplos. $\mathrm{Na}$ fase de encerramento o arquivamento de lições aprendidas, condução de entrevistas pós-projeto, testes e avaliações de conhecimentos, transferência pessoal de conhecimentos e experiências para outros times de projetos e a checagem de metas de aprendizagem, formam outros exemplos.

Diante do exposto, as práticas podem ser entendidas ainda como sendo inter projetos entre diversos projetos - e, intraprojetos - ocorrendo no âmbito do próprio projeto. Diferenciais competitivos observados em organizações projetizadas, de acordo com Shinoda, Maximiano e Sbragia (2015).

Para Nonaka e Takeuchi (1997), fatores influenciadores podem ser intenções, autonomia na gestão e utilização do conhecimento, flutuação e caos criativo, redundâncias e variedades de requisitos. Von Krogh, Ichijo e Nonaka (2001), utilizam capacitadores do conhecimento como influenciadores, como instalar a visão do conhecimento, gerenciar conversas, mobilizar ativistas do 
Um estudo sobre gestão do conhecimento em escritórios de gerenciamento de projetos na região metropolitana de Belo Horizonte Vladimir Alexei Rodrigues Rocha, Ricardo Rodrigues Barbosa, George Leal Jamil

conhecimento e globalizar o conhecimento. Koskinen e Pihlanto (2008) destacam fatores que afetam o compartilhamento do conhecimento em um contexto de projetos, como o papel do líder, motivação para compartilhar o conhecimento, atitude de se preocupar com o outro, cultura organizacional e de projeto, valores e crenças.

Prencipe, Brady, Marshall e Tell (2005) discutem alguns fatores influenciadores que dizem ser condições que definem a zona de manobra em que se dá a evolução da aprendizagem interprojeto. São eles: complexidade técnica, novidade técnica, duração do projeto, porte da organização, estilo da organização do projeto e alocação de pessoal.

Desse modo, os fatores influenciadores podem ser compreendidos de forma mais ampla, perpassando por cultura, estrutura, papeis, processos e ferramentas organizacionais, além dos fatores individuais, gerando impacto nas práticas de gestão do conhecimento.

\subsection{Gestão do Conhecimento em Escritórios de Gerenciamento de Projetos}

Uma organização que trabalha com projetos, em função de sua importância, tamanho e estratégia, utiliza de uma área específica para realizar sua gestão. Esse tipo de área é conhecido como Escritório de Gerenciamento de Projetos (EGP) e tem a responsabilidade de manter toda a produção intelectual relativa à gestão de projetos (KERZNER, 2006). Por convenção, o PMI define um Escritório de Gerenciamento de Projetos (EGP) como sendo uma estrutura organizacional que padroniza os processos de governança relacionados a projetos, e facilita o compartilhamento de recursos, metodologias, ferramentas e técnicas (PMI, 2017).

Para se mensurar o desempenho dos projetos, Fatores Críticos de Sucesso (FCS) são levados em consideração como uma espécie de representação prática da execução de projetos, segundo Oliveira, Cruz e Oliveira (2018). Projetos classificados como bem sucedidos, possuem FCS específicos, definidos no início da gestão dos projetos e variam de acordo com a necessidade da organização. Envolvem, desde melhores práticas identificadas em um projeto, passando por estratégias, táticas, operações, métodos de condução de projetos, até ações envolvendo motivação da equipe. O Escritório de Gerenciamento de Projetos (EGP) é responsável pela organização dos recursos empregados em cada projeto, além do alinhamento entre a estratégia organizacional e o seu desdobramento nos múltiplos setores em que os projetos atuam. 
Os resultados, parciais ou finais de um projeto, positivos ou não, são armazenados e produzem o que se convencionou chamar de "lições aprendidas". Segundo Rocha e Aganette (2017), esses registros fazem parte da base de conhecimento. Essa base é administrada, tanto para armazenar, quanto recuperar informações, em Sistemas Integrados de Gestão (SIG), nos Escritórios de Gerenciamento de Projetos. Dessa forma, as experiências registradas e utilizadas em novos projetos, podem representar alguma vantagem competitiva na gestão dos projetos a serem desenvolvidos.

Koskinen (2010) acrescenta que empresas com estrutura de gestão de projetos são organizações nas quais a maior parte dos produtos é feita sob medida para os clientes. Para fins de entendimento no presente estudo, os escritórios de projetos trabalham com a gestão dos recursos alocados no desenvolvimento dos produtos de forma original, sob medida, de acordo com a temporalidade acordada com clientes internos e externos.

As organizações com estruturas projetizadas têm desafios a serem vencidos para realizar a gestão de seus conhecimentos. Turner (2005) colabora com essa afirmação quando diz que ser capaz de gerenciar o conhecimento de gestão de projetos, ser capaz de lembrar como entregar projetos com êxito e melhorar esse conhecimento, é crucial para o sucesso da organização.

Além disso, em empresas projetizadas a gestão do conhecimento pode enfrentar problemas, com novos conhecimentos criados em projetos paralelos e não utilizados em outros projetos. Esse aspecto é também discutido por Pinto (2005), ao questionar como se pode institucionalizar e tornar essas práticas sistemáticas, quando em sua própria natureza a gestão de projetos representa um empreendimento único. São algumas lacunas e desafios para a gestão da organização.

Organizações projetizadas apresentam lacunas, segundo alguns autores. Koskinen e Pihlanto (2008) apontam algumas fraquezas das empresas projetizadas: falha no aprendizado organizacional, dificuldade de conectar os projetos aos processos de negócio da organização, falta de tempo para reflexão durante a implementação dos projetos em função do cronograma e constante falta de recursos.

\subsection{Estudo anteriores}

Alguns estudos refletem a preocupação de pesquisadores brasileiros em lidar com a gestão do conhecimento em organizações que utilizam a gestão de projetos. Shinoda, Maximiano e Sbragia 
Um estudo sobre gestão do conhecimento em escritórios de gerenciamento de projetos na região metropolitana de Belo Horizonte Vladimir Alexei Rodrigues Rocha, Ricardo Rodrigues Barbosa, George Leal Jamil

(2015), realizaram uma pesquisa caracterizada como sendo de natureza qualitativa, exploratória e descritiva.

Segundo os autores, as iniciativas de gestão do conhecimento em organizações que utilizam gestão de projetos são relativamente novas, à época da pesquisa. Além disso, quando se trata de gestão do conhecimento em organizações projetizadas, como aquelas pesquisadas pelos autores, os impactos na temporalidade e singularidade do projeto tornam-se um desafio ainda maior.

Os resultados alcançados por Shinoda, Maximiano e Sbragia (2015), que pesquisaram em três organizações que prestam serviços de consultoria (interna ou externa), apontam para a criação de um contexto favorável como elemento relevante para uma gestão eficaz do conhecimento em projetos. Resultado similar ao encontrado na presente pesquisa, realizada em Escritórios de Gerenciamento de Projetos, estrutura responsável pela gestão do conhecimento nas quatro organizações pesquisadas.

Outro estudo sobre gestão do conhecimento em projetos foi desenvolvido por Oliveira, Cruz e Oliveira (2018). Os autores dedicaram atenção aos mecanismos de mensuração do desempenho de um Escritório de gerenciamento de Projetos, a partir de Fatores Críticos de Sucesso (FCS). A pesquisa levou em consideração aspectos da gestão empresarial como "estratégia", que comporta aspectos de metodologia de gestão de projetos e estrutura organizacional; "pessoas", em que os FCS abordados foram a motivação e a importância da capacitação; e "operações", com a estabilidade do ambiente de projetos, qualidade, auditoria e os entregáveis do projeto. Os fatores descritos, guardam relação com conhecimentos relevantes e fatores facilitadores na presente pesquisa. Observa-se, com relativa frequência, a existência de práticas similares com nomenclaturas próprias, variando de organização para organização.

Com abordagem voltada para a execução do projeto, a fim de evidenciar a importância do conhecimento como um "insumo estratégico", Rocha e Aganette (2017) pesquisaram Lições Aprendidas em projetos. Trata-se do registro das ocorrências consideradas relevantes em um projeto. As ocorrências podem ser tanto resultados positivos como cumprimento de prazos e cronogramas, quanto resultados negativos como atrasos e outras intercorrências. Os autores, por meio da aplicação de uma survey online, reforçam o conceito de Lição Aprendida como diferencial na gestão de projetos, assim como também na gestão do conhecimento. As lições aprendidas, foram registradas na presente pesquisa como conhecimentos relevantes apontados pelos entrevistados. 
Um estudo sobre gestão do conhecimento em escritórios de gerenciamento de projetos na região metropolitana de Belo Horizonte Vladimir Alexei Rodrigues Rocha, Ricardo Rodrigues Barbosa, George Leal Jamil

Nota-se que os estudos anteriores registram componentes que se relacionam com conhecimentos relevantes, fatores facilitadores e um contexto favorável à gestão do conhecimento organizacional, sobretudo em Escritórios de Gerenciamento de Projetos.

\section{Procedimentos metodológicos}

Para se alcançar os objetivos propostos na introdução do estudo, adotou-se uma abordagem qualitativa, de natureza descritiva, tendo como parâmetros os modelos adotados por Shinoda, Maximiano e Sbragia (2015) em organizações projetizadas, e adaptado em Rocha (2017), para organizações com Escritórios de Gerenciamento de Projetos.

O método adotado foi o estudo de múltiplos casos e pode ser classificado como integrado, pois envolve mais de uma unidade de análise. Para Yin (2015), o estudo de caso é o método adequado quando as questões "como" e "por que" são propostas; o investigador tem pouco controle sobre os eventos; e o enfoque está sobre um fenômeno contemporâneo da vida real.

As fontes de evidências utilizadas no estudo foram: entrevistas, documentação e observação direta, conforme Tabela 2.

Tabela 2 - Entrevista, documentação e observação direta

\begin{tabular}{|c|c|c|c|}
\hline Técnica & Definição & Ponto forte & Ponto fraco \\
\hline Entrevista & $\begin{array}{l}\text { Coletas de dados, } \\
\text { informações e } \\
\text { evidências para } \\
\text { compreender o } \\
\text { significado que os } \\
\text { entrevistados } \\
\text { atribuem às questões, } \\
\text { situações e contextos } \\
\text { apresentados pelo } \\
\text { pesquisador. }\end{array}$ & $\begin{array}{l}\text { Direcionador: permite } \\
\text { foco nos tópicos do } \\
\text { estudo de caso; } \\
\text { Perceptível: fornece } \\
\text { possibilidades para } \\
\text { inferências diante das } \\
\text { explanações recebidas. }\end{array}$ & $\begin{array}{l}\text { Parcialidade: em função } \\
\text { da má formulação das } \\
\text { perguntas aos } \\
\text { entrevistados; } \\
\text { Incorreções: em função } \\
\text { da obtenção de } \\
\text { informações a partir da } \\
\text { memória dos } \\
\text { entrevistados; } \\
\text { Reflexividade: quando o } \\
\text { entrevistado responde de } \\
\text { forma a satisfazer o } \\
\text { entrevistador, e não } \\
\text { conforme o ocorrido. }\end{array}$ \\
\hline Documentação & $\begin{array}{l}\text { Utilização de } \\
\text { procedimentos, } \\
\text { processos, imagens, }\end{array}$ & $\begin{array}{l}\text { Rastreabilidade: contém } \\
\text { referência exata de um } \\
\text { evento; }\end{array}$ & $\begin{array}{l}\text { Recuperabilidade: pode } \\
\text { ser difícil encontrar } \\
\text { determinado documento; }\end{array}$ \\
\hline
\end{tabular}


Um estudo sobre gestão do conhecimento em escritórios de gerenciamento de projetos na região metropolitana de Belo Horizonte Vladimir Alexei Rodrigues Rocha, Ricardo Rodrigues Barbosa, George Leal Jamil

\begin{tabular}{|c|c|c|c|}
\hline & $\begin{array}{l}\text { filmes, cronogramas, } \\
\text { pareceres, mensagens } \\
\text { e demais tipos de } \\
\text { documentos }\end{array}$ & $\begin{array}{l}\text { Alcance: pode cobrir } \\
\text { longos períodos de } \\
\text { tempo e diversos } \\
\text { eventos; } \\
\text { Consolidado: pode ser } \\
\text { revisto. }\end{array}$ & $\begin{array}{l}\text { Parcialidade: por parte de } \\
\text { quem produziu o } \\
\text { documento; } \\
\text { Acesso: pode ser negado } \\
\text { pela organização }\end{array}$ \\
\hline $\begin{array}{l}\text { Observação } \\
\text { direta }\end{array}$ & $\begin{array}{l}\text { Envolve a percepção } \\
\text { do observador. } \\
\text { Procedimento } \\
\text { empírico, de natureza } \\
\text { sensorial, que permite } \\
\text { a coleta de dados em } \\
\text { determinadas } \\
\text { situações. }\end{array}$ & $\begin{array}{l}\text { Realidade: cobre } \\
\text { eventos em tempo real; } \\
\text { Contextual: cobre o } \\
\text { contexto do caso. }\end{array}$ & $\begin{array}{l}\text { Tempo: consome mais } \\
\text { tempo; } \\
\text { Seletividade: ampla } \\
\text { cobertura é mais difícil; } \\
\text { Reflexividade: ambiente } \\
\text { pode mudar com a } \\
\text { presença do observador. }\end{array}$ \\
\hline
\end{tabular}

Fonte: adaptado de MARCONI; LAKATOS, 2010; MARTINS; THEÓFILO, 2009; YIN, 2015.

É possível alavancar os pontos fortes e, ciente dos pontos fracos, mitigá-los no planejamento da pesquisa. Para isso, nas entrevistas, foi possível utilizar o benefício de serem direcionadas, por meio de uma explicação detalhada dos objetivos da pesquisa, e dos tópicos que se desejava estudar junto aos entrevistados. Os pontos fracos das entrevistas foram atenuados a partir do envio prévio, por mensagem eletrônica (e-mail), do roteiro daquilo que seria abordado na entrevista. Além disso, procurou-se manter postura de imparcialidade durante a entrevista de forma a reduzir a reflexibilidade.

Em relação aos documentos, foi possível manter contato com os entrevistados, por mensagem eletrônica após as entrevistas, caso algum documento não fosse apresentado durante as entrevistas, permitindo continuidade na análise dos documentos, conforme definido na tabela 2. Por fim, as observações diretas ocorreram em ambiente reservado pelos entrevistados, de forma a que só permaneceram os envolvidos na pesquisa (entrevistador e entrevistado).

O caráter da pesquisa é analítico. Segundo Nakano e Fleury (2005), o caráter analítico é voltado para a elaboração de conceitos e construtos que permitem a descrição e a análise do fenômeno, uma vez que o problema da pesquisa envolve compreender como as organizações realizam a gestão do conhecimento em Escritórios de Gerenciamento de Projetos.

A amostra da pesquisa foi escolhida por conveniência e composta por quatro organizações e seus respectivos Escritórios de Gerenciamento de Projetos (EGP). Cada um dos estudos de caso respeitaram os seguintes critérios: organizações brasileiras, que possuem Escritórios de 
Gerenciamento de Projetos, cujo líder do EGP possua mais de um ano de empresa em posição de liderança.

A decisão por selecionar organizações brasileiras adveio do interesse em estudar práticas desenvolvidas no país. Existem organizações que importam a gestão do conhecimento da matriz no exterior. Tais procedimentos podem possuir diversos níveis de maturidade, diferente daqueles correspondentes às organizações brasileiras.

A escolha por Escritórios de Gerenciamento de Projetos, está relacionada às dificuldades provenientes da natureza dos projetos para a gestão do conhecimento, abordado na introdução da pesquisa (DAVENPORT \& PRUSAK, 1998). As dificuldades podem ser resumidas na temporalidade - os projetos são concluídos e nem sempre os conhecimentos gerados são integrados ao conhecimento organizacional - e a singularidade, já que cada projeto é único.

O critério objetivo definido para escolha dos entrevistados, foi que o entrevistado deveria ter, no mínimo, um ano de organização em cargo de liderança, pois, segundo Hanisch et al. (2009), o conhecimento em projetos possui forte influência do líder.

Em cada uma das organizações estudadas, contou-se com a interlocução de profissionais em cargo de coordenação, gerência, superintendência e diretoria. Em alguns Escritórios de Gerenciamento de Projetos, as atribuições eram semelhantes, porém, os cargos diferentes.

A coleta e a análise de dados envolveram o desenho de um protocolo do estudo de caso, realização dos estudos de caso individuais por meio de entrevistas, análise individual e posterior análise integrada dos casos.

O protocolo do estudo de caso, segundo Yin (2015), é elemento importante para orientar o pesquisador na realização da coleta de dados, aumentando a confiabilidade da pesquisa. Adotou-se como protocolo um documento contendo visão geral da pesquisa, procedimentos da coleta de dados, questões formuladas para o estudo dos casos e um guia para a produção da análise dos dados obtidos.

As entrevistas foram previamente combinadas, confirmadas por mensagens eletrônicas (emails) e ocorreram no período de outubro de 2016 a abril de 2017, com algumas remarcações ao longo do período em função da agenda dos entrevistados. Consistiram em uma apresentação inicial dos objetivos da pesquisa e os tópicos propostos para a discussão. Levantou-se o perfil do entrevistado, tempo de organização, posto ocupado, bem como as práticas de gestão do 
conhecimento desenvolvidos em suas respectivas organizações. Todas as entrevistas foram gravadas com autorização dos entrevistados.

A análise de conteúdo trata-se de um conjunto de técnicas de análise de comunicações que utiliza procedimentos sistemáticos e objetivos de descrição do conteúdo das mensagens, indicadores - quantitativos ou qualitativos -, que permitam a inferência de conhecimentos relativos às condições de produção das mensagens. O tratamento dos dados ocorreu de forma qualitativa baseado mais na presença do que na frequência da aparição (BARDIN, 2008). A exploração do material coletado envolveu seleção, análise, inferência e interpretação dos dados em cada caso estudado individualmente. Em seguida realizou-se a análise integrada, aplicando o mesmo procedimento de exploração do material coletado em cada caso estudado individualmente.

Os estudos de caso seguiram a mesma estrutura: visão geral, resultados coletados, conhecimentos relevantes, fatores influenciadores, práticas de gestão do conhecimento intra e interprojetos (ROCHA, 2017), apresentando a análise integrada dos casos.

As organizações foram caracterizadas da seguinte forma:

Empresa A - segmento de tecnologia com 400 colaboradores diretos. Atua com linhas de produtos de solução de software para vários segmentos, incluindo a própria área de Tecnologia da Informação (TI). Média de 35 projetos ocorrendo simultaneamente em diversas fases - iniciação, planejamento, execução, monitoramento e controle e encerramento. O Escritório de Gerenciamento de Projetos é vinculado à diretoria de TI. É conduzido por profissional com mais de sete anos em cargos de liderança.

Empresa B - mercado financeiro. Conta com 680 colaboradores com atuação em várias cidades do país. Possui, em fases diversas, 200 projetos, sendo que destes, $60 \mathrm{em}$ fase final de entrega. O EGP está vinculado à Governança de TI, centralizando todos os projetos, independentemente de sua natureza. Seu gestor conta com mais de seis anos de experiência no cargo.

Empresa C - prestadora de serviços de abastecimento de água, esgotamento sanitário e resíduos sólidos. A partir de 2008 criou a diretoria de projetos e consequentemente seu EGP. Realizou em torno de 380 projetos, de grande e pequeno portes. O líder entrevistado possuía mais de trinta anos de empresa, sendo oito diretamente no EGP.

Empresa D - Organização nacional, com polo em Minas Gerais, pertencente ao terceiro setor. Os projetos gerenciados pelo EGP são classificados como administrativos e financeiros. 
Um estudo sobre gestão do conhecimento em escritórios de gerenciamento de projetos na região metropolitana de Belo Horizonte Vladimir Alexei Rodrigues Rocha, Ricardo Rodrigues Barbosa, George Leal Jamil

Administra 30 projetos, em fases diferentes, com duração média em torno de dois anos. A liderança contava dois anos à frente do grupo vinculado ao EGP.

As práticas intraprojeto tiveram como referência o processo de gestão do conhecimento de recuperação, criação/aquisição, compartilhamento e armazenamento. Fleury e Fleury (2004), propõem como síntese dos processos de Gestão do Conhecimento: aquisição e desenvolvimento de conhecimentos, disseminação e construção da memória organizacional que também foram considerados.

Para efeito de apresentação dos resultados, serão descritos no próximo tópico a análise integrada obtida nas quatro organizações pesquisadas.

\section{Resultados e discussões}

Os casos estudados possuem como características comuns, serem organizações brasileiras e com estrutura de gestão centralizada de projetos, que são os Escritórios de Gerenciamento de Projetos. Por outro lado, se diferenciam tanto nas características gerais, quanto em seus setores de atuação e suas dimensões. Em todos os casos, os profissionais entrevistados possuíam cargos de liderança e o entrevistado com menor tempo de organização, contava com dois anos.

\subsection{Conhecimentos relevantes}

A partir da análise integrada dos casos, foi possível identificar oito tipos de conhecimentos relevantes para as organizações participantes do estudo, conforme descrito na Tabela 3.

Tabela 3 - Conhecimentos relevantes identificados

\begin{tabular}{|c|l|}
\hline $\begin{array}{c}\text { TIPOS DE } \\
\text { CONHECIMENTO }\end{array}$ & \multicolumn{1}{c|}{ DESCRIÇÃO } \\
\hline Processos de gestão & $\begin{array}{l}\text { Métodos e modelos documentados para desenvolvimento das } \\
\text { atividades dos projetos }\end{array}$ \\
\hline Negócio & $\begin{array}{l}\text { Tipos de produtos e serviços vinculados ao core business de cada } \\
\text { organização }\end{array}$ \\
\hline Documentação técnica & $\begin{array}{l}\text { Documentos vinculados ao desenvolvimento dos produtos, Know } \\
\text { how aplicado em cada projeto e seu histórico de execução }- \text { lições } \\
\text { aprendidas }\end{array}$ \\
\hline Experiência & $\begin{array}{l}\text { Histórico dos projetos (lições aprendidas), atuação em diversos } \\
\text { projetos com escopos similares, atuação em projetos com }\end{array}$ \\
\hline
\end{tabular}




\section{IRIS)}

Um estudo sobre gestão do conhecimento em escritórios de gerenciamento de projetos na região metropolitana de Belo Horizonte Vladimir Alexei Rodrigues Rocha, Ricardo Rodrigues Barbosa, George Leal Jamil

\begin{tabular}{|l|l|}
\hline & $\begin{array}{l}\text { escopos diferentes, capacidade de articulação junto a stakeholders } \\
\text { na solução de problemas para alcance de metas e resultados } \\
\text { planejados }\end{array}$ \\
\hline Comunicação & $\begin{array}{l}\text { Compreensão clara das tarefas desempenhadas por cada ator } \\
\text { envolvido, assim como os demais influenciadores nos resultados do } \\
\text { projeto e consequentemente na gestão do conhecimento }\end{array}$ \\
\hline Conhecimento Técnico & $\begin{array}{l}\text { Visão sistêmica de todos os processos, compreensão técnica dos } \\
\text { diversos recursos necessários para a consolidação das práticas e } \\
\text { técnicas vinculadas aos projetos }\end{array}$ \\
\hline $\begin{array}{l}\text { Estudo de viabilidade dos } \\
\text { projetos }\end{array}$ & $\begin{array}{l}\text { Definição de critérios seguindo diretrizes estratégicas da } \\
\text { organização para seleção de projetos, uma vez que os recursos } \\
\text { são finitos e para cada cliente seu projeto é prioritário }\end{array}$ \\
\hline Modelo de gestão & $\begin{array}{l}\text { Conhecimento das atividades do projeto, construção de padrões } \\
\text { que permitam que as práticas adotadas sejam repetidas e } \\
\text { melhoradas continuamente }\end{array}$ \\
\hline
\end{tabular}

Fonte: autores, 2017.

Quanto a relevância do conhecimento, é oportuno retomar uma teoria utilizada por Fleury e Fleury (2004), que evidencia a diferença existente entre conhecimento e competência. Conhecimento está relacionado ao que é preciso saber para realizar uma tarefa. No entanto, só o conhecimento não é suficiente para realiza-la. Seria necessário, ainda segundo os autores, habilidades que definem como agir e atitude (querer agir). O estudo se ateve ao conhecimento.

Esses conhecimentos foram apontados pelos entrevistados como relevantes nas organizações pesquisadas. Podem ser categorizados de forma a constituir grupos de conhecimentos. Categorizam-se em: conhecimentos de gestão - processos de gestão, modelo de gestão, negócio, comunicação e experiência, e conhecimentos técnicos - documentação técnica, conhecimento técnico e estudo de viabilidade dos projetos. Esse tipo de conhecimento, possui partes tácitas e explícitas, por isso podem ser adquiridos por meio de treinamentos estruturados - para os aspectos codificáveis do conhecimento - ou por meio de capacitação no próprio ambiente de trabalho, aplicável aos aspectos mais tácitos, que só aprendem por meio da experiência, conforme se depreende de Nonaka e Takeuchi (1997).

A estrutura desenvolvida por Hanisch et al (2009), descrita no referencial teórico desse estudo (conhecimento em projetos, conhecimento sobre projetos e conhecimento entre projetos), encontra-se categorizada nesse estudo como conhecimento de gestão - sobre projetos e entre projetos - e conhecimentos técnicos (em projetos).

\subsection{Fatores influenciadores - facilitadores}


Um estudo sobre gestão do conhecimento em escritórios de gerenciamento de projetos na região metropolitana de Belo Horizonte Vladimir Alexei Rodrigues Rocha, Ricardo Rodrigues Barbosa, George Leal Jamil

Percebeu-se a existência de fatores que influenciam a forma de se realizar a Gestão do Conhecimento. São influenciadores, por exemplo, o porte da organização, uma vez que esse aspecto influencia o grau de alinhamento entre o Escritório de Gerenciamento de Projetos e as equipes executoras - quando as atividades do projeto não ocorrem apenas no EGP.

O porte das organizações parece influenciar o nível de formalização dos processos e a sua estrutura de documentação dos conhecimentos produzidos. Em duas organizações com porte menor, dentre as quatro pesquisadas, existem tendências de se buscar o conhecimento diretamente com a pessoa que atuou em determinada tarefa, o que sugere um alto grau de informalidade. A informalidade, segundo os entrevistados, permite que as atividades sejam mais céleres e menos "burocráticas". Por outro lado, reconhecem o risco maior de se perder informações e conhecimentos.

Os fatores identificados, nas organizações pesquisadas, como influenciadores facilitadores na Gestão do Conhecimento foram descritos na tabela 4:

Tabela 4 - Fatores facilitadores

\begin{tabular}{|c|c|}
\hline FATORES & $\begin{array}{l}\text { DE QUE MANEIRA FACILITAM A GESTÃO DO } \\
\text { CONHECIMENTO }\end{array}$ \\
\hline Estrutura organizacional & $\begin{array}{l}\text { Existência nas organizações de um Setor de projetos, Escritório de } \\
\text { Gerenciamento de Projetos, Superintendência de Projetos e } \\
\text { Governança Corporativa como estruturas matriciais fortes e } \\
\text { projetizadas. }\end{array}$ \\
\hline Metodologia & $\begin{array}{l}\text { Processos estruturados, documentos do empreendimento, fluxo de } \\
\text { demandas, estudos de viabilidade, complexidade dos projetos, } \\
\text { operação em células, mobilidade da equipe }\end{array}$ \\
\hline Sistema & $\begin{array}{l}\text { Biblioteca de projetos e plataformas virtuais de gestão dos projetos, } \\
\text { melhorando a comunicação e minimizando impactos da distância } \\
\text { física, quando aplicável }\end{array}$ \\
\hline Segmento do negócio & $\begin{array}{l}\text { Intensidade da concorrência, cultura organizacional, busca contínua } \\
\text { por inovação, sazonalidade, podem influenciar os projetos }\end{array}$ \\
\hline Experiência & $\begin{array}{l}\text { Vivência dos profissionais na condução dos projetos, conseguindo } \\
\text { articular equipes na recuperação, criação, compartilhamento e } \\
\text { armazenamento dos conhecimentos adquiridos na gestão dos } \\
\text { projetos }\end{array}$ \\
\hline
\end{tabular}

Fonte: autores, 2017.

Parte dos fatores facilitadores registrados no estudo, podem surgir nas organizações com nomes diferentes, influenciados pela cultura, entretanto, atuando com os mesmos propósitos, apresentados também como conhecimentos relevantes, em alguns casos. Outros fatores comuns, 
Um estudo sobre gestão do conhecimento em escritórios de gerenciamento de projetos na região metropolitana de Belo Horizonte Vladimir Alexei Rodrigues Rocha, Ricardo Rodrigues Barbosa, George Leal Jamil

identificados em estudos anteriores (SHINODA, MAXIMIANO \& SBRAGIA, 2015), podem ser citados como comunicação, cultura organizacional e maturidade dos processos.

Os sistemas computadorizados são considerados pelos entrevistados como imprescindíveis e aliados na disseminação do conhecimento, principalmente nas organizações cujo produto/serviço é de tecnologia da informação. Tais sistemas influenciam na integração dos atores e processos, permitindo maior acompanhamento dos resultados, tanto na gestão do projeto quanto no desenvolvimento do produto nas relações entre projetos.

\subsection{Fatores influenciadores - dificultadores}

Os fatores dificultadores para a Gestão do Conhecimento identificados a partir da análise dos casos foram consolidados na tabela 5 .

Tabela 5 - Fatores dificultadores

\begin{tabular}{|l|l|}
\hline \multicolumn{1}{|c|}{ FATORES INFLUENCIADORES } & \multicolumn{1}{|c|}{$\begin{array}{c}\text { DE QUE MANEIRA DIFICULTAM A } \\
\text { GESTÃO DO CONHECIMENTO }\end{array}$} \\
\hline Resistência à cultura documental & $\begin{array}{l}\text { Na organização, manuseio e arquivamento dos } \\
\text { documentos }\end{array}$ \\
\hline Não priorização da gestão do conhecimento & $\begin{array}{l}\text { Na recuperação, criação, compartilhamento e } \\
\text { arquivamento do conhecimento }\end{array}$ \\
\hline $\begin{array}{l}\text { Falta de integração entre as áreas envolvidas } \\
\text { no projeto }\end{array}$ & $\begin{array}{l}\text { No cumprimento dos prazos previstos nos } \\
\text { cronogramas de atividades dos projetos }\end{array}$ \\
\hline Rotatividade de pessoal & $\begin{array}{l}\text { Na preservação de informações e } \\
\text { conhecimentos difundidos nos projetos }\end{array}$ \\
\hline
\end{tabular}

Fonte: autores, 2017.

Apesar do número inferior de fatores dificultadores, a intensidade e a frequência com que foram observados considerou-se relevante, porque esses fatores permeiam as operações de toda a organização, com reflexos na gestão do conhecimento nos Escritórios de Gerenciamento de Projetos.

A resistência à prática de se registrar, documentar, manter atualizada e consultar a documentação dos projetos, faz com que o conhecimento se concentre nas pessoas da organização, dificultando o trabalho de integração do Escritório de Gerenciamento de Projetos.

A falta de integração entre as áreas, na execução dos projetos, dificulta o compartilhamento de conhecimento, conforme observado nas empresas estudadas. Fenômeno que ocorre, tanto para 
Um estudo sobre gestão do conhecimento em escritórios de gerenciamento de projetos na região metropolitana de Belo Horizonte Vladimir Alexei Rodrigues Rocha, Ricardo Rodrigues Barbosa, George Leal Jamil

aqueles que possuem EGP em diferentes localidades, assim como nas organizações em que há um EGP central.

Os EGP pesquisados, reconheceram a importância da gestão do conhecimento, todavia, processos estruturados, documentados, sistemas alimentados e retroalimentados adequadamente foram considerados insuficientes, ante a rotina de gestão dos projetos, por sua temporalidade e originalidade.

\subsection{Práticas intraprojeto de Gestão do Conhecimento}

As práticas que são realizadas ao longo de um projeto, consideradas intraprojeto, ou seja, práticas de gestão adotadas na preparação, iniciação, planejamento, monitoramento e controle e encerramento do projeto, foram destacadas na tabela 6 :

Tabela 6 - Práticas de Gestão do Conhecimento intraprojeto

\begin{tabular}{|c|c|c|c|}
\hline \multicolumn{4}{|c|}{ INTRAPROJETO } \\
\hline RECUPERAÇÃO & $\begin{array}{l}\text { CRIAÇÃO / } \\
\text { AQUISIÇÃO }\end{array}$ & COMPARTILHAMENTO & ARMAZENAMENTO \\
\hline $\begin{array}{l}\text { - Registros de projetos } \\
\text { anteriores (histórico, } \\
\text { lições aprendidas) } \\
\text { •Comitês de conclusão } \\
\text { e reuniões de início } \\
\text { de projetos } \\
\text { - Sistema de gestão de } \\
\text { projetos online } \\
\text { - Gestor e colaborador } \\
\text { de projetos } \\
\text { experientes } \\
\text {-Passagem de "bastão" } \\
\text { entre as etapas na } \\
\text { célula de trabalho } \\
\text {-Procedimentos } \\
\text { operacionais } \\
\text {-Diretrizes da } \\
\text { Diretoria }\end{array}$ & $\begin{array}{l}\text {-Planejamento do } \\
\text { projeto } \\
\text { - Detalhamento do } \\
\text { plano do projeto } \\
\text { - Treinamentos } \\
\text { - Participação de } \\
\text { especialistas } \\
\text { (internos e } \\
\text { externos) em } \\
\text { novos produtos } \\
\text { - Fóruns criados } \\
\text { pelo EGP } \\
\text { - Reuniões técnicas }\end{array}$ & $\begin{array}{l}\text {-Planejamento do projeto } \\
\text { (Termo de Abertura do } \\
\text { Projeto) } \\
\text { Status Report- } \\
\text { acompanhamento do } \\
\text { estado do projeto } \\
\text { Disponibilização do projeto } \\
\text { no sistema para consulta e } \\
\text { acompanhamento } \\
\text { - Treinamentos (on the job) } \\
\text {-Comunicações com os } \\
\text { clientes } \\
\text {-Conversas informais } \\
\text { - Pesquisa de satisfação } \\
\text { • Fórum de lançamento do } \\
\text { projeto } \\
\text { - Kickoff com a presença do } \\
\text { cliente } \\
\text { •Interação com o cliente } \\
\text { •Interação com equipe de } \\
\text { execução física }\end{array}$ & $\begin{array}{l}\text {-Templates desenvolvidos } \\
\text { e adotados como } \\
\text { default (padrão) } \\
\text { - Registro de lições } \\
\text { aprendidas de acordo } \\
\text { com os projetos } \\
\text { - Materiais } \\
\text { disponibilizados na } \\
\text { rede sobre o projeto } \\
\text {-Membros da equipe } \\
\text { Gestores do projeto e } \\
\text { do Escritório de } \\
\text { Projetos } \\
\text { - Relatório final } \\
\text {-Materiais gerados no } \\
\text { projeto } \\
\text {-Memórias das reuniões } \\
\text { registradas no sistema } \\
\text { de gestão }\end{array}$ \\
\hline
\end{tabular}

Fonte: autores, 2017. 
Percebeu-se que algumas práticas se repetiram nas organizações, modificando-se a nomenclatura e, por isso, foram suprimidas do quadro. Junto às práticas comuns, provenientes, em parte, pelo uso das melhores práticas difundidas pela Associação de Projetos PMI (Project Management Institute), observam-se iniciativas ainda embrionárias nas organizações pesquisadas. Apesar de contar com tempo de existência das organizações pesquisadas.

\subsection{Práticas interprojetos de Gestão do Conhecimento}

As práticas de gestão interprojetos identificadas nos casos estudados foram informadas na tabela 7 . Percebeu-se, pelas entrevistas, a importância do planejamento e encadeamento das ações dos projetos (causa e efeito) e o quanto a metodologia é valorizada pelas lideranças, como "caminho para percorrer" na identificação de gargalos, mitigação de riscos e solução de problemas.

Tabela 7 - Práticas interprojetos de Gestão do Conhecimento

\begin{tabular}{|c|c|c|}
\hline $\begin{array}{l}\text { ARMAZENAMENTO / } \\
\text { RECUPERAÇÃO }\end{array}$ & CRIAÇÃO / AQUISIÇÃO & COMPARTILHAMENTO \\
\hline $\begin{array}{l}\text { - Metodologia de Gerenciamento de } \\
\text { Projetos } \\
\text { - Escritório de Gerenciamento de } \\
\text { Projetos (EGP) } \\
\text { - Sistema de registro dos projetos }\end{array}$ & $\begin{array}{l}\text { Rituais periódicos de } \\
\text { acompanhamento } \\
\text { Procedimentos Operacionais } \\
\text { Caracterizações dos } \\
\text { empreendimentos }\end{array}$ & $\begin{array}{l}\text { •Comunicações estruturadas } \\
\text { •Comunicações informais } \\
\text { - Produção de material de apoio a } \\
\text { projetos (modelos) }\end{array}$ \\
\hline
\end{tabular}

Fonte: autores, 2017.

\section{Considerações finais}

O problema que estimulou a pesquisa, gerando seu objetivo - como ocorre a Gestão do Conhecimento em projetos realizados em organizações brasileiras -, foi respondido neste estudo a partir do mapeamento dos conhecimentos relevantes, análise dos fatores influenciadores e análise da aplicação das práticas de gestão do conhecimento desenvolvidas nos quatro escritórios de gerenciamento de projetos pesquisados.

Pode-se inferir como uma das principais conclusões após a realização da pesquisa que o contexto organizacional favorável - e, neste sentido, o favorável seriam processos estruturados, 
colaboradores capacitados e avaliados continuamente no resgate, criação, compartilhamento e armazenamento - seria o ponto mais relevante para o aprimoramento da gestão do conhecimento.

Outra observação, diz respeito a algum possível "destaque" quanto à Gestão do Conhecimento. Conclui-se, neste trabalho, à semelhança de trabalhos anteriores (SHINODA, MAXIMIANO \& SBRAGIA, 2015), que o aspecto importante do Conhecimento a ser gerenciado varia conforme o contexto, isto é, de acordo com as características e objetivos de cada projeto. Variam também os tipos de Conhecimento considerados relevantes pelos entrevistados, como pode ser comparado com o trabalho desenvolvido por Shinoda, Maximiano \& Sbragia (2015), mantendo como desafio a temporalidade e a originalidade dos projetos (PMI, 2017).

Foi possível observar também que a Gestão do Conhecimento contribui para a Gestão de Projetos por meio da sistematização dos seus processos, e quando estruturada formalmente, favorece o reaproveitamento de conhecimentos já possuídos, provocando sensível aumento da eficiência e, consequentemente, do aprendizado. Por outro lado, a gestão de projetos também beneficia a gestão do conhecimento por ser uma prática em expansão que auxilia na solução de problemas e novos negócios.

Percebeu-se ainda que o tempo dedicado à Gestão do Conhecimento é baixo, sendo justificado pelos entrevistados em função do volume de projetos e dos prazos exíguos para execução.

Como recomendações para estudos futuros, pode-se testar a hipótese, de que as organizações percebem como benefícios, nas práticas de gestão intra e inter projetos, apenas o armazenamento dos conhecimentos, comparando-os com recuperação, compartilhamento e criação/aquisição. Outro ponto relevante pode ser ampliar a amostra em outras pesquisas, já que a adotada neste estudo foi por conveniência. Buscar replicar o estudo com uma amostra maior, permitiria maior generalização dos resultados encontrados.

Por fim, cabe destacar alguns aprendizados auferidos ao longo da pesquisa. A presença de profissionais experientes na implantação e condução da metodologia de gerenciamento de projetos, com liderança consolidada pelo aprendizado - sucesso e insucesso - em outros projetos e empreendimentos, favorecem a preservação e disseminação do conhecimento nas organizações que lidam com gestão de projetos ou possuem Escritórios de Gerenciamento de Projetos. 


\section{IRIS)}

Um estudo sobre gestão do conhecimento em escritórios de gerenciamento de projetos na região metropolitana de Belo Horizonte Vladimir Alexei Rodrigues Rocha, Ricardo Rodrigues Barbosa, George Leal Jamil

\section{Referências}

AUBRY, M.; MÜLLER, R. GLÜCKLER, J. Exploring PMOs through community of practice theory. Project Management Journal, Vol. 42, pp. 42-56, 2011.

BAHIA, F. O PMO e a Gerência do Conhecimento. In: BARCAUI, André (Organizador). PMO Escritórios de Projetos, Programas e Portfólios na prática. Brasport Livros e Multimídia Ltda., Rio de Janeiro, 2012.

BARDIN, L. Análise de Conteúdo. 1.ed., Lisboa: Edições 70, 280 p., 2008.

BESEN, F.; TECCHIO, E.; FIALHO, F. A. P. Liderança Autêntica e a gestão do conhecimento. Revista Gestão \& Produção, São Carlos, SP, v.24, no.1, pp.2-14, 2017.

DALKIR, K. Knowledge Management and practice. Oxford: England: Elsevier, 2005.

DAVENPORT, T. H.; PRUSAK, L. Conhecimento Empresarial: como as organizações gerencial o seu capital intelectual. Rio de Janeiro, Campus, 2008.

DRUCKER, P. F. Administrando em tempos de grandes mudanças. São Paulo: Pioneira, 1995.

DRUCKER, P. F. The new realities. New York: Transaction publishers, 2011.

FAUCHER, J.-B. P. L.; EVERETT, A. M.; LAWSON, R. Reconstituting knowledge management. Journal of Knowledge Management. Vol. 12, no. 3, pp. 3-16, 2008.

FLEURY, A.; FLEURY, M. T. L. Estratégias empresariais e a formação de competências. São Paulo: Atlas, 2004.

GONÇALVES, A. P. F. Gestão do Conhecimento em Projetos. Dissertação de Mestrado, Instituto Universitário de Lisboa, Portugal, 2016.

HANISCH, B.; LINDNER, F.; MUELLER A.; WALD, A. Knowledge Management in project environments. Journal of Knowledge Management, vol.13, n.4, p.148-160, 2009.

HARALDSDOTTIR, R. K.; GUNNLAUGSDOTTIR, J.; HVANNBERG, E. T.; CHRISTENSEN, P. H. Registration, access and use of personal knowledge in organizations. International Journal of Information Management. Vol.40, 0268-4012, pp.8-16, 2018.

HORNSTEIN, H. A. The integration of project management and organizational change management is now a necessity. International Journal od Project Management. JPMA-01682; 0263-7863, Elsevier Ltd., 2014.

JANNUZZI, C. S. C.; FALSARELLA, O. M.; SUGAHARA, C. R. Gestão do Conhecimento: um estudo de modelos e sua relação com inovação nas organizações. Perspectiva em Ciência da Informação, v.21, n.1, p.97-118, 2016.

KERZNER, H. Gestão de Projetos: As melhores práticas. Porto Alegre: Bookman, 2006.

KOSKINEM, K. U.; PIHLANTO, P. Knowledge Management in Project-Based Companies: An Organic Perspective. Hampshire: Palgrave Macmillan, 2008. 
KOSKINEM, K. U. Recursive view of the Project-based companies knowledge production. Journal of Knowledge Management, vol.14, n.2, 2010.

MARCONI, M. A.; LAKATOS, E. M. Fundamentos de Metodologia Científica. 7.ed.

São Paulo: Atlas, 2010.

MARTINS, G. A.; THEÓFILO, C. R. Metodologia da Investigação Científica para Ciências Sociais Aplicadas. 2.ed. São Paulo: Atlas, 2009.

MÊGNIGBÊTO, E. Correlation Between Transmission Power and Some Indicators Used to Measure the Knowledge-Based Economy: Case of Six OECD Countries. J Knowl Econ 9, 1168-1183, 2018.

MENEZES, L. Benefícios do PMO. In: BARCAUI, André (Organizador). PMO Escritórios de Projetos, Programas e Portfólios na prática. Brasport Livros e Multimídia Ltda., Rio de Janeiro, 2012.

MÜLLER, R.; GLÜCKLER, J.; AUBRY, M. Project Management Knowledge Flows in Networks of Project Managers and Project Management Offices: A Case Study in the Pharmaceutical Industry. Project Management Journal, Vol. 44, pp. 4-19, 2013.

NADAE, J.; CARVALHO, M. M. A Knowledge Management perspective of the Project Management Office. Brazilian Journal of Operations \& Production Management. São Paulo, vol.14, n.3, pp. 350-362, 2017.

NAKANO, D. N.; FLEURY, A. C. C. Conhecimento organizacional: uma revisão conceitual de modelos e quadros de referência. Revista Produto \& Produção. Porto Alegre, vol.8, n.2, p.11-23, 2005.

NONAKA, I.; TAKEUCHI, H. Criação de conhecimento na empresa: como as empresas japonesas geram a dinâmica da inovação. Rio de Janeiro: Campus, 1997.

NONAKA, I.; TAKEUCHI, H. Gestão do Conhecimento. Porto Alegre: Bookman, 2008.

NORTH, K. Gestão do Conhecimento: um guia prático rumo à empresa inteligente. Rio de Janeiro: QualityMark, 2010.

OCHIENG, O. M.; NYANGOSI, R. Effect of Knowledge Management Practices on micro, small and medium enterprises performance in Migori County. Africa International Journal of Management, Education and Governance, 2017.

OLIVEIRA, R. R.; CRUZ, J. E.; OLIVEIRA, R. R. Fatores Críticos de Sucesso na gestão de projetos: análise dos indicadores que constituem os predecessores da estratégia, pessoas e operações. Revista de Gestão e Projetos, v. 9, n. 3, 2018.

PINTO, J. K.; Forewords. In: LOVE, P.; FONG, P.; IRANI, Z. Management of Knowledge in Project Environments. Oxford: England, Elsevier, 2005.

PMI. Project Management Institute. Um guia do conhecimento em gerenciamento de projetos (Guia PMBOK). 5.ed. Livro em versão eletrônica. Pensilvânia, 2013. 
PRENCIPE, A.; BRADY, T.; MARSHALL, N.; TELL, F. Making sense of learning landscapes in Projectbased organizations. In: LOVE, P. E. D. (Org.). Management of Knowledge in Project Environments. Oxford: Elsevier, 2005.

ROCHA, D. B.; AGANETTE, E. C. Lições aprendidas: elo entre gestão do conhecimento e gestão de projetos. Anais do XVII Encontro Nacional de Pesquisa em Ciência da Informação - ENANCIB, Marília, SP, 2017.

ROCHA, V. A. R. Gestão do Conhecimento em projetos: um estudo de múltiplos casos em organizações brasileiras. Dissertação de Mestrado. Faculdade IETEC, Belo Horizonte, Minas Gerais, 2017.

SAMPAIO, R. R.; ROSA, C. P.; PEREIRA, H. B. B.; Mapeamento dos fluxos de informação e conhecimento: a governança de TI sob a ótica das redes sociais. Revista Gestão \& Produção. São Carlos, SP, v.19, no.2, pp. 377-387, 2012.

SANTOS, R. B. P.; ISATON, C.; JUNGLES, A. E. Fatores de influência na troca de conhecimento: um estudo de caso em uma construtora de médio porte. $9^{\circ}$ Simpósio Brasileiro de Gestão e Economia da Construção, $6^{\circ}$ Encontro Latino-Americano de Gestão e Economia da Construção promovido pela Associação Nacional de Tecnologia do Ambiente Construído (ANTAC), São Carlos, SP, Brasil, 2015.

SERRAT, O. Managing Knowledge in project environments. Knowledge Solutions Book. Asian Development Bank, Springer, Singapore, pp. 509-522, 2017.

SHINODA, A. C. M.; MAXIMIANO, A. C. A.; SBRAGIA, R. Gestão do Conhecimento em organizações orientadas para projetos. Revista de Gestão e Projetos. Vol.6, n.1, 2015.

SVEIBY, K. E. The new organizational wealth: managing and measuring Knowledge based assets. San Francisco: Berett-Koehler Publichers, 1997.

TREVISAN, L. C.; DAMIAN, I. P. M. Gestão do Conhecimento: diretrizes e práticas recomendadas às organizações. Revista Ciência da Informação, Brasília, DF, v.47, no.2, pp.21-34, 2018.

TURNER, J. R. Forewords. In: LOVE, P.; FONG, P.; IRANI, Z. Management Knowledge in Project Environments. Oxford: England, Elsevier, 2005.

VON KROGH, G.; NONAKA, I.; ICHIJO, K. Facilitando a criação de conhecimento: reinventando a empresa com poder da inovação contínua. Rio de Janeiro: Campus, 2001.

YIN, R. K. Estudo de Caso: Planejamento e Métodos. Porto Alegre: Bookman, 2015.

Data de submissão: 28 de maio de 2020

Data de aceitação: 20 de outubro de 2020 